\title{
The isoperimetric problem for lens spaces
}

\author{
Celso Viana ${ }^{1}$ (D) \\ Received: 15 December 2017 / Revised: 3 September 2018 / Published online: 13 December 2018 \\ (c) The Author(s) 2018
}

\begin{abstract}
We solve the isoperimetric problem in the lens spaces with large fundamental group. Namely, we prove that the isoperimetric surfaces are either geodesic spheres or quotients of Clifford tori. We also show that the isoperimetric problem in the lens spaces $L(3,1)$ and $L(3,2)$ follows from the proof of the Willmore conjecture by Marques and Neves.
\end{abstract}

\section{Introduction}

The isoperimetric problem is a classical subject in Differential Geometry with its origin in ancient Greece. It consists in finding on a Riemannian manifold $M$ the regions that minimize the perimeter among sets enclosing the same volume. The solutions are called isoperimetric regions and their boundaries isoperimetric hypersurfaces. The Euclidean plane is historically the first space where the problem started to be investigated rigorously. It is now a well known fact that the round circles are the optimal curves for the problem. This geometric fact is often seen through the following classical inequality:

$$
L^{2} \geq 4 \pi A
$$

where $L$ and $A$ stand for the length and the enclosed area of a simple closed curve $\gamma: \mathbb{S}^{1} \rightarrow \mathbb{R}^{2}$ respectively.

The framework of geometric measure theory and its tools work successfully well in tackling the aspects of existence and regularity of this variational problem. When $M^{n+1}$ is closed or homogeneous, then isoperimetric hypersurfaces do exist and are smooth up to a closed set of Hausdorff dimension $n-7$. The regular part is a stable hypersurface of constant mean curvature. This major contribution to the isoperimetric problem was achieved thanks to the efforts of many people, including Almgren [2],

\footnotetext{
Communicated by F.C. Marques.

$凶$ Celso Viana

celso.viana.14@ucl.ac.uk

1 University College London UCL Union Building, 25 Gordon Street, London WC1E 6BT, UK
} 
Schoen and Simon [33], and others (see [20] for a comprehensive list). Despite the long history of the problem, it remains largely open with few 3-manifolds where the problem is completely understood.

The simply connected space forms, $\mathbb{S}^{n+1}, \mathbb{R}^{n+1}$ and $\mathbb{H}^{n+1}$, are the most appealing spaces to begin the study of the isoperimetric problem. It turns out that their symmetries are enough to characterize the geodesic spheres as the isoperimetric hypersurfaces, see [8].

A complete solution on $\mathbb{S}^{2} \times \mathbb{S}^{1}$ with the standard product metric can be found in Ref. [25]. For other homogeneous manifolds with certain product structure, such as $\mathbb{H}^{2} \times \mathbb{R}, \mathbb{H}^{2} \times \mathbb{S}^{1}$, and $\mathbb{S}^{n} \times \mathbb{R}$, see [16,24,25] respectively. The case $\mathbb{S}^{1} \times \mathbb{R}^{n}$ is also treated in Ref. [25] and they show that when $n \geq 9$ unduloids are minimizers rather than cylinders for certain volumes. One key idea exploited in the results listed above is the use of symmetry to reduce the problem to an ODE analysis. The case $T^{2} \times \mathbb{R}$ where $T^{2}$ is a flat torus is not solved in full; great progress can be found in Refs. [14,27,29]. More generally, it is known that boundaries of small isoperimetric regions in closed manifolds are nearly round spheres, see [22,23]. To finish this brief and not exhaustive account of results on the isoperimetric problem we mention that Bray and Morgan [6,7] classified the horizon homologous isoperimetric surfaces in the Schwarzschild manifold. The works $[6,12]$ highlight the interesting relationship between isoperimetric surfaces and the concept of mass in general relativity.

We will be interested in spherical space forms in this paper. A significant result in this direction was given in Ref. [28] where Ros and Ritoré solved the isoperimetric problem in the projective space $\mathbb{R P}^{3}$. They show that the solutions are geodesic spheres or flat tori. Later, Ros [31] used the above result to give a proof of the Willmore conjecture in $\mathbb{S}^{3}$ for the special case of surfaces that are invariant by the antipodal map.

The real projective space is a special case of an important family of Riemannian manifolds, namely the lens spaces $L(p, q)$. These are spherical space forms obtained as a quotient of $\mathbb{S}^{3}$ by a finite group of isometries that are isomorphic to $\mathbb{Z}_{p}$ but which also depend on $q$. They are, along with $\mathbb{S}^{2} \times \mathbb{S}^{1}$, characterized by having Heegaard genus one. One of the few results concerning the isoperimetric problem in the lens spaces was given in Ref. [21] where the authors provide Steiner and Schwarz symmetrization for certain fiber bundles including $L(p, q)$. This work together with [25] implies that the minimal Clifford torus in the lens spaces $L(p, 1)$ is isoperimetric for every $p \geq 2$. This implication does not readily apply to $L(p, q)$ when $q \neq 1$, see Sect. 3 for further discussion.

We give a complete solution for the isoperimetric problem in the lens spaces with large fundamental group:

Theorem 1.1 There exists a positive integer $p_{0}$ such that for every $p \geq p_{0}$ and every $q \geq 1$ the isoperimetric surfaces in $L(p, q)$ are either geodesic spheres or quotient of Clifford tori.

The isoperimetric problem in dimension three was previously solved for only a finite number of non-diffeomorphic 3-manifolds.

We also add to the literature the observation that the proof of the Willmore conjecture by Marques and Neves [19] can be applied to extend the work of Ros and Ritoré [28], on the classification of stable cmc surfaces in $\mathbb{R P}^{3}$, to $L(3,1)$ and $L(3,2)$ : 
Theorem 1.2 The immersed stable cmc surfaces in $L(3,1)$ and $L(3,2)$ are either geodesic spheres or quotient of Clifford tori. Moreover, the minimal Clifford torus is, up to ambient isometries, the only index one minimal surface in $L(3,1)$ and $L(3,2)$.

Theorem 1.2 in the particular case of isoperimetric surfaces in $L(3,1)$ appeared first in [32, Theorem 15].

The idea of the proof of Theorem 1.1 is as follows. Stability implies that every isoperimetric surface is connected and its genus is $0,1,2$ or 3 . It follows from a classical result of Hopf that if the genus is zero, then it is a geodesic sphere. From [28] we know that if the genus is 1 , then it is flat, and this forces the surface to be a quotient of a Clifford tori. We are left to rule out other topological types. To do so we argue by contradiction. We assume that there exists a sequence of lens spaces with increasing fundamental group containing isoperimetric surfaces of genus 2 or 3 . After a suitable rescaling on the metrics we use compactness results to obtain a limit for the sequence of lens spaces which will be a flat three manifold of rank one. In the same way, the sequence of isoperimetric surfaces will converge to a flat surface in the respective ambient manifold. The topology of the surfaces will force the limit to be an union of planes. On the other hand, the minimization property rules this configuration out. This argument works in a wide class of lens spaces. For the remaining cases, we change the strategy and use varifold convergence applied to the pullback surfaces in the round three sphere.

The arguments in the proof of Theorem 1.1 generalize naturally for the Berger spheres $\mathbb{S}_{\varepsilon}^{3}$. This is a well known one parameter family of homogeneous metrics on the 3 -sphere. The parameter $\varepsilon$ measures the size of the Hopf fibers; the case $\varepsilon=$ 1 corresponds to the round metric. When $\varepsilon$ is not too small, spheres are the only solutions of the isoperimetric problem. When $\varepsilon$ is very small, some tori are better candidates to solve the isoperimetric problem rather than spheres for certain volumes. By considering sequences of Berger spheres $\mathbb{S}_{\varepsilon}^{3}$ with $\varepsilon \rightarrow 0$, we prove:

Theorem 1.3 There exists $\varepsilon_{0}>0$ such that for every $\varepsilon<\varepsilon_{0}$ the isoperimetric surfaces in the Berger spheres $\mathbb{S}_{\varepsilon}^{3}$ are either rotationally invariant spheres or tori.

\section{Preliminaries}

Let $\left(M^{n+1}, g\right)$ be an orientable Riemannian manifold of dimension $n+1$. The $n+1$ dimensional Hausdorff measure of a region $\Omega \subset M$ is denoted by $|\Omega|$. Similarly, we denote the $n$-dimensional Hausdorff measure of the hypersurface $\partial \Omega \subset M$ by $|\partial \Omega|$. The class of regions considered here are those of finite perimeter, see [34].

\subsection{Isoperimetric}

A region $\Omega \subset M$ is called an Isoperimetric region if

$$
|\partial \Omega|=\inf \left\{\left|\partial \Omega^{\prime}\right|: \Omega^{\prime} \subset M \text { and }\left|\Omega^{\prime}\right|=|\Omega|\right\} \text {. }
$$

In this case, the hypersurface $\Sigma=\partial \Omega$ is called an Isoperimetric hypersurface. 
The existence of isoperimetric hypersurfaces is, in general, handled by a compactness theorem from geometric measure theory. For non-compact manifolds one needs to be careful since a minimizing sequence of regions of fixed volume may drift off to infinity. We recommend [20] for a recent reference on the regularity of isoperimetric hypersurfaces:

Theorem 2.1 Let $\left(M^{n+1}, g\right)$ be a closed Riemannian manifold. For every $0<t<$ $\operatorname{vol}(M)$ there exists an isoperimetric region $\Omega$ satisfying $|\Omega|=t$. Moreover, $\Sigma=\partial \Omega$ is smooth up to a closed set of Hausdorff dimension $n-7$.

\subsection{Stability}

Isoperimetric hypersurfaces are stable critical points of the area functional for variations that preserve the enclosed volume; thus, the regular part of isoperimetric hypersurfaces has constant mean curvature. More generally, we say that a two-sided isometric immersion $\phi: \Sigma^{n} \rightarrow M^{n+1}$ has constant mean curvature $(\mathrm{cmc})$ if it is a critical point of the area functional for volume preserving variations. A critical points is called stable cmc if the second derivative of the area is non-negative for such variations. Equivalently, $\phi$ is stable cmc if for every $f \in C^{\infty}(\Sigma)$ with compact support such that $\int_{\Sigma} f d_{\Sigma}=0$, we have

$$
I(f, f)=-\int_{\Sigma} f L f d_{\Sigma}:=\int_{\Sigma}|\nabla f|^{2}-\left(\operatorname{Ric}(N, N)+|A|^{2}\right) f^{2} d_{\Sigma} \geq 0 .
$$

$N$ is an unit normal vector field along $\Sigma$ and $A$ is the second fundamental form of the immersion $\phi$. The mean curvature of $\Sigma$, denoted by $H$, is defined by $2 H=\operatorname{trace}(A)$.

The study of stable cmc hypersurfaces started in Refs. $[3,4]$ with a new characterization of the geodesic spheres in the simply connected space forms $\mathbb{R}^{n+1}, \mathbb{S}^{n+1}$ and $\mathbb{H}^{n+1}$. The classification of stable cmc surfaces is often a way to approach the isoperimetric problem in reasonable spaces. With this purpose in mind, Ros and Ritoré [28] used the Hersch-Yau trick to study orientable stable cmc surfaces on three manifolds of positive Ricci curvature.

Theorem 2.2 (Ros-Ritoré [28]) Let $(M, g)$ be a three manifold with positive Ricci curvature. If $\phi: \Sigma \rightarrow M^{3}$ is a compact (two-sided) stable cmc immersion, then $g(\Sigma) \leq 3$. Moreover, if $g(\Sigma)=2$ or 3 , then

$$
\left(\frac{1}{2} \inf _{\Sigma} \operatorname{Ric}_{M}+H^{2}\right)|\Sigma| \leq 2 \pi
$$

Proposition 2.3 Let $\phi: \Sigma \rightarrow M^{3}$ be a stable cmc immersion with constant mean curvature $H$ into an spherical space form $M=\mathbb{S}^{3} / G$. Then

(1) If $g(\Sigma)=2$ or 3 , then $\left(1+H^{2}\right)|\Sigma| \leq 2 \pi$.

(2) If $g(\Sigma)=2$ or 3 and $|G| \leq 4$, then $\phi$ is an embedding. Moreover, if $|G| \leq 6$, then the pullback of $\Sigma$, through the covering map $\Pi: \mathbb{S}^{3} \rightarrow M^{3}$, is connected.

(3) If $|G|=2$ or 3 , then $g(\Sigma)=0$ or 1 . 
Proof The first statement follows from the previous theorem since $\operatorname{Ric}_{M}=2$. Let $\phi_{*}: \pi_{1}(\Sigma) \rightarrow \pi_{1}(M)$ be the induced map in fundamental groups. As $K=\operatorname{Ker}\left(\phi_{*}\right)$ has finite index there exists a finite covering $\psi: \widetilde{\Sigma} \rightarrow \Sigma$ such that $\operatorname{Im}\left(\psi_{*}\right)=K$ and $(\phi \circ \psi)_{*}=0$. This means there exists a lifting of this map into $\mathbb{S}^{3}$ and we denote it by $\widetilde{\phi}: \widetilde{\Sigma} \rightarrow \mathbb{S}^{3}$. It follows that $\left(1+H^{2}\right)|\widetilde{\Sigma}| \leq|G| 2 \pi$. If $\phi$ is not an embedding, then $\widetilde{\phi}$ is not embedding either. By the work of Li and Yau [18] the Willmore energy of the immersed surface $\widetilde{\Sigma}$, i.e. $\mathcal{W}(\widetilde{\Sigma})=\int_{\widetilde{\Sigma}}\left(1+H^{2}\right) d v l_{\Sigma}$, is strictly greater ${ }^{1}$ than $8 \pi$. Therefore, if $|G| \leq 4$, we obtain a contradiction and $\widetilde{\phi}$ is an embedding. Moreover, for closed surfaces with genus greater than or equal to 1 in $\mathbb{S}^{3}$ the Willmore conjecture, recently proved in Ref. [19], states that $\mathcal{W}(\Sigma) \geq 2 \pi^{2}$. Let $\cup_{i=1}^{l} \widetilde{\Sigma}_{i}$ be the pre-image of $\Sigma$ by the universal covering map, then

$$
2 l \pi^{2} \leq \sum_{i=1}^{l} \mathcal{W}\left(\widetilde{\Sigma}_{i}\right)=|G| \mathcal{W}(\Sigma) \leq|G| 2 \pi \Rightarrow \frac{|G|}{l} \geq \pi
$$

Therefore, if $|G| \leq 6$, then $l=1$ and $|G| \geq \pi$. In particular, if $|G|=2$ or 3 , then there exist no stable cmc surface $\Sigma$ with $g(\Sigma) \geq 2$.

Definition 2.2 For each $r \in\left(0, \frac{\pi}{2}\right)$ we define the Clifford Torus $T_{r}$ as:

$$
T_{r}=\mathbb{S}^{1}(\cos (r)) \times \mathbb{S}^{1}(\sin (r)) \subset \mathbb{S}^{3}
$$

Every flat torus with constant mean curvature in $\mathbb{S}^{3}$ is congruent to the Clifford torus $T_{r}$ of same mean curvature. This follows from the Rigidity Theorem, pg. 49 in Ref. [9], for these surfaces have the same second fundamental form.

Corollary 2.4 The stable cmc surfaces in $L(3,1)$ and $L(3,2)$ are totally umbilical spheres or flat tori. In addition, the index one minimal surfaces in $L(3,1)$ and $L(3,2)$ are congruent to the projection of minimal Clifford torus.

Proof Let $\Sigma \subset L(3, q), q=1,2$, be in the conditions of the corollary. By Proposition 2.3, $g(\Sigma)=0$ or 1 . If $g(\Sigma)=0$, then it follows from the Hopf holomorphic quadratic differential that $\Sigma$ is totally umbilical. If $g(\Sigma)=1$, then it is proved in Ref. [28] that $\Sigma$ is flat.

\subsection{Isoperimetric profile}

The isoperimetric properties of $M$ can be encapsulated in a single function called the isoperimetric profile. This is the function $I_{M}:[0, \operatorname{vol}(M)] \rightarrow[0,+\infty)$ defined by

$$
I_{M}(v)=\inf \{|\partial \Omega|: \Omega \subset M \text { and }|\Omega|=v\} .
$$

We finish the section with some well known facts on the analytic nature of $I_{M}$. These will be used later in Sect. 3 .

\footnotetext{
1 The case $\mathcal{W}(\Sigma)=8 \pi$ is discussed in Ref. [28].
} 
Let $\Omega$ be an isoperimetric region in $M$ such that $|\Omega|=v$ for some $v \in(0, \operatorname{Vol}(M))$. The function $I_{M}$ has left and right derivatives $\left(I_{M}\right)_{-}^{\prime}(v)$ and $\left(I_{M}\right)_{+}^{\prime}(v)$. In addition, if $H$ is the mean curvature of $\Sigma=\partial \Omega$ in the direction of the inward unit vector, then

$$
\left(I_{M}\right)_{+}^{\prime}(v) \leq 2 H \leq\left(I_{M}\right)_{-}^{\prime}(v)
$$

The second derivative also exists but weakly in the sense of comparison functions. More precisely, we say $f^{\prime \prime} \leq h$ weakly at $x_{0}$ if there exists a smooth function $g$ such that $f \leq g, f\left(x_{0}\right)=g\left(x_{0}\right)$, and $g^{\prime \prime} \leq h$. In this sense we have

$$
I_{M}(v)^{2} I_{M}^{\prime \prime}(v)+\int_{\Sigma}\left(\operatorname{Ric}_{g}(N, N)+|A|^{2}\right) d_{\Sigma} \leq 0 .
$$

The Eqs. (2.5) and (2.6) are first presented on [5] (see also Section 5 in Ref. [15]). We sketch the proof of (2.5) and (2.6).

Let $\Sigma_{V}$ be the variation $\Sigma_{t}=\exp _{\Sigma}(t N)$ of $\Sigma$ reparametrized in terms of the enclosed volume $v(t)$. In addition, let $\phi(t)(\operatorname{resp} . \phi(v))$ be the area of $\Sigma_{t}$ (resp. $\left.\Sigma_{v}\right)$. By the first variation formula for the area and volume we have $\phi^{\prime}(0)=2 H|\Sigma|$ and $v^{\prime}(0)=|\Sigma|$ respectively. Since $\phi^{\prime}(t)=\phi^{\prime}(v) v^{\prime}(t)$, we conclude that $\phi^{\prime}(v(0))=2 H$ and also that $v^{\prime}(0)^{2} \phi^{\prime \prime}(v(0))=\phi^{\prime \prime}(0)-\phi^{\prime}(v(0)) v^{\prime \prime}(0)$. On the other hand, the second derivative of area for general variations implies the following:

$$
\begin{aligned}
\phi^{\prime \prime}(0) & =-\int_{\Sigma} 1 L 1 d_{\Sigma}+2 H v^{\prime \prime}(0) \\
& =-\int_{\Sigma}\left(\operatorname{Ric}_{g}(N, N)+|A|^{2}\right) d_{\Sigma}+2 H v^{\prime \prime}(0)
\end{aligned}
$$

Hence, in the sense of comparison functions, (2.6) follows from:

$$
\phi(v(0))^{2} \phi^{\prime \prime}(v(0))+\int_{\Sigma}\left(\operatorname{Ric}_{g}(N, N)+|A|^{2}\right) d_{\Sigma}=0 .
$$

\section{The Isoperimetric problem in the lens Spaces}

In order to define the lens spaces, we first recall the round three sphere as:

$$
\mathbb{S}^{3}=\left\{(z, w) \in \mathbb{C}^{2}:|z|^{2}+|w|^{2}=1\right\}
$$

Fix $p, q$ integers with the following property $1 \leq q<p$ and $\operatorname{gcd}(p, q)=1$. Let $\mathbb{Z}_{p}$ be the group $\mathbb{Z} / p \mathbb{Z}$ acting on $\mathbb{S}^{3}$ as follows:

$$
m \in \mathbb{Z}_{p} \mapsto m \cdot(z, w)=\left(e^{\frac{2 \pi i q m}{p}} z, e^{\frac{2 \pi i m}{p}} w\right) .
$$

The group $\mathbb{Z}_{p}$ acts freely and properly discontinuously on $\mathbb{S}^{3}$. The orbit space $\mathbb{S}^{3} / \mathbb{Z}_{p}$ is a closed three manifold called the Lens space, it is denoted by $L(p, q)$. 
The Hopf fibration, which is the Riemannian submersion $h: \mathbb{S}^{3} \rightarrow \mathbb{S}^{2}\left(\frac{1}{2}\right)=$ $\mathbb{C} \cup\{\infty\}$ defined by $h(z, w)=\frac{z}{w}$, can be extended naturally to $L(p, q)$. Indeed, the group $\mathbb{Z}_{p}$ acts on the set of Hopf fibers through the cyclic action of $\Gamma_{p}=\left\langle e^{\frac{2 \pi i(q-1)}{p}}\right\rangle$ on $\mathbb{S}^{2}\left(\frac{1}{2}\right)$ given by

$$
e^{\frac{2 \pi i(q-1)}{p}}: \mathbb{C} \longrightarrow \mathbb{C}, \quad \lambda \longmapsto e^{\frac{2 \pi i(q-1)}{p}} \cdot \lambda
$$

The Hopf fibration for $L(p, q)$ is then defined as $h: L(p, q) \rightarrow \mathbb{S}^{2}(1 / 2) / \Gamma_{p}$. The set $\mathbb{S}^{2}(1 / 2) / \Gamma_{p}$ is a two dimensional orbifold with conical singularities at the north and south pole when $q \neq 1$. The pre-image of these singularities are called the critical fibers of $h$.

The preimage of horizontal slices of $\mathbb{S}^{2}(1 / 2) / \Gamma_{p}$ via $h$ corresponds to the Clifford torus described in (2.3). They are natural candidates to solve the isoperimetric problem in $L(p, q)$.

\subsection{Comments on Steiner symmetrization for lens spaces}

Steiner and Schwarz symmetrization theorems were proved in Ref. [21] for certain fiber bundles including lens spaces $L(p, q)$. To highlight the application of this symmetrization procedure to the isoperimetric problem for lens spaces, we restrict to the case $L(p, 1)$ where the Hopf fibration $h: L(p, 1) \rightarrow \mathbb{S}^{2}\left(\frac{1}{2}\right)$ is a smooth Riemannian submersion.

The symmetrization consists of associating to each set of finite perimeter $R \subset$ $L(p, 1)$ the set $\operatorname{Sym}(R)$ in the product manifold $\mathbb{S}^{2}\left(\frac{1}{2}\right) \times \mathbb{S}^{1}\left(\frac{1}{p}\right)$ defined by replacing the slice of $R$ in each fiber with a ball of the same volume about the respective fiber in the product. The coarea formula for Riemannian submersions implies that $\operatorname{Sym}(R)$ and $R$ have the same volume. It is proved in Ref. [21] that $\operatorname{Sym}(R)$ has no greater perimeter than $R$.

One immediate consequence is that $I_{L(p, 1)} \geq I_{\mathbb{S}^{2}\left(\frac{1}{2}\right) \times \mathbb{S}^{1}\left(\frac{1}{p}\right)}$. Applying the classification of the isoperimetric problem on $\mathbb{S}^{2}\left(\frac{1}{2}\right) \times \mathbb{S}^{1}\left(\frac{1}{p}\right)$, [25], we conclude that $I_{L(p, 1)}=I_{\mathbb{S}^{2}\left(\frac{1}{2}\right) \times \mathbb{S}^{1}\left(\frac{1}{p}\right)}$ in a interval around $V=\frac{\operatorname{Vol}(L(p, 1))}{2}$. In particular, the minimal Clifford torus is isoperimetric in $L(p, 1)$ for every $p \geq 3$. The isoperimetric profiles, however, do not coincide as the profile of geodesic spheres on the respective spaces are different. Therefore, this technique is not enough to completely solve the isoperimetric problem.

It is also important to point out that, for general lens spaces $L(p, q)$, there is no analogue of [25] for $\mathbb{S}^{2}\left(\frac{1}{2}\right) / \Gamma_{p} \times \mathbb{S}^{1}\left(\frac{1}{n_{p}}\right)$ which is a manifold having co-dimension two singularities. 


\subsection{Some aspects of lens spaces}

For every $x \in L(p, q)$ the injectivity radius of $L(p, q)$ at $x$ satisfies $\operatorname{inj}_{x} L(p, q) \geq \frac{\pi}{p}$, with equality only at points in the critical fibers. Indeed, for $\theta=e^{\frac{2 \pi i}{p}}$ we have:

$$
\begin{gathered}
a^{2}:=d_{\mathbb{R}^{4}}^{2}\left[\left(\theta^{k q} z, \theta^{k} w\right),(z, w)\right]=\left|\theta^{k q}-1\right|^{2}|z|^{2}+\left|\theta^{k}-1\right|^{2}|w|^{2} \geq|\theta-1|^{2} \\
d_{\mathbb{S}^{3}}\left[\left(\theta^{k q} z, \theta^{k} w\right),(z, w)\right]=2 \arcsin \left(\frac{a}{2}\right) \geq 2 \arcsin \left(\frac{2}{2} \sin \frac{2 \pi}{2 p}\right)=\frac{2 \pi}{p} .
\end{gathered}
$$

However, is not true in general that $\operatorname{inj}(L(p, q), x)=O\left(\frac{1}{p}\right)$. Here, the notation $i n j_{x} L(p, q)=O(\tau)$ means that $\frac{1}{C} \tau \leq i n j_{x} L(p, q) \leq C \tau$, for a constant $C$ which is independent of $p, q$ and $x \in L(p, q)$.

Example 3.1 Let us consider $L\left(k^{2}, k+1\right), k \in \mathbb{Z}_{+}$. We show that the injectivity radius at points far away from the critical fibres are $O\left(\frac{1}{k}\right)$. If the round metric is scaled by the factor $k^{2}$, then we have the Riemannian submersion:

$$
h:\left(L\left(k^{2}, k+1\right), k^{2} g_{0}, x_{k}\right) \rightarrow\left(\mathbb{S}^{2} / \mathbb{Z}_{k}, k^{2} g_{\mathbb{S}^{2}}, h\left(x_{k}\right)\right)
$$

The fibers have constant length $2 \pi$ except the critical fibres which have length $\frac{2 \pi}{p}$. The right hand side will converge, as $k \rightarrow \infty$, to $\mathbb{S}^{1} \times \mathbb{R}$. It follows from the coarea formula for Riemannian submersions that the volume of the geodesic ball $B_{4 \pi}\left(x_{k}\right)$ in $\left(L\left(k^{2}, k+1\right), k^{2} g_{0}, x_{k}\right)$ is bounded from below. Therefore, by Cheeger's inequality, Lemma 51 in Ref. [26], the injectivity radius of the sequence $\left(L\left(k^{2}, k+1\right), k^{2} g_{0}, x_{k}\right)$ is bounded from below. This sequence converges to a flat $T^{2} \times \mathbb{R}$.

If $x, y \in T_{\frac{\pi}{4}} / \mathbb{Z}_{p} \subset L(p, q)$, then $d_{L(p, q)}(x, y) \geq C d_{T_{\frac{\pi}{4}} / \mathbb{Z}_{p}}(x, y)$ for some constant $C>0$ independent of $p, q$. Thus intrinsic and extrinsic distances on $T_{\frac{\pi}{4}} / \mathbb{Z}_{p}$ are equivalent.

Lemma 3.2 If $x \in T_{\frac{\pi}{4}} / \mathbb{Z}_{p} \subset L(p, q)$ and the extrinsic diameter of $T_{\frac{\pi}{4}} / \mathbb{Z}_{p}$ in $L(p, q)$ is bounded from below, then $\operatorname{inj}_{x} L(p, q)=O\left(\frac{1}{p}\right)$.

Proof Let $\lambda_{p}=\frac{1}{i n j_{x} L(p, q)}$ and recall that $\frac{1}{\lambda_{p}} \geq \frac{\pi}{p}$. Without loss of generality, let's assume that diameter ${ }_{L(p, q)}\left(T_{\frac{\pi}{4}} / \mathbb{Z}_{p}\right) \geq 1$. Hence, under the rescaled metric $\lambda_{p}^{2} g_{\mathbb{S}^{3}}$, the extrinsic diameter of $T_{\frac{\pi}{4}} / \mathbb{Z}_{p}$ is greater than or equal to $\lambda_{p}$. Let $\gamma_{p}(t)$ be a geodesic segment realizing the intrinsic diameter of $T_{\frac{\pi}{4}} / \mathbb{Z}_{p}$. Thus, we can find disjoint balls $B_{R}\left(x_{i}\right) \subset L(p, q)$, with $R<\frac{C}{2}, x_{i} \in \gamma_{p}(t)$, and $i=1, \ldots,\left[\lambda_{p}\right]+1$. Hence,

$$
\sum_{i=1}^{\left[\lambda_{p}\right]+1} \mathcal{H}^{2}\left(B_{R}\left(x_{i_{0}}\right) \cap T_{\frac{\pi}{4}} / \mathbb{Z}_{p}\right) \leq\left|T_{\frac{\pi}{4}} / \mathbb{Z}_{p}\right|=\lambda_{p}^{2} \frac{2 \pi^{2}}{p}
$$


Therefore, there exists $i_{0} \in\left\{1, \ldots,\left[\lambda_{p}\right]+1\right\}$ such that:

$$
C_{1} \leq \mathcal{H}^{2}\left(B_{R}\left(x_{i_{0}}\right) \cap T_{\frac{\pi}{4}} / \mathbb{Z}_{p}\right) \leq \frac{2 \pi^{2} \cdot \lambda_{p}}{p} .
$$

The first inequality follows from the Monotonicity Formula, Proposition 6.1 in the Appendix, applied to $T_{\frac{\pi}{4}} \subset\left(L(p, q), \lambda_{p}^{2} g_{\mathbb{S}^{3}}, x\right)$. This finishes the proof of the lemma.

Let's use the notation $\mathbb{Z}_{p}^{q}$ to represent the group $\mathbb{Z}_{p}$ acting on $\mathbb{S}^{3}$ and its dependence on the parameter $q$. By using the toroidal coordinate system for $\mathbb{S}^{3}$,

$$
\varphi_{r}: \mathbb{R}^{2} \rightarrow T_{r}: \varphi_{r}(u, v)=\left(\cos (r) e^{2 \pi i u}, \sin (r) e^{2 \pi i v}\right) \in \mathbb{S}^{3}
$$

the action of $\mathbb{Z}_{p}^{q}$ on $T_{r}$ corresponds to the following action on $\mathbb{R}^{2}$ :

$$
(u, v) \longmapsto\left(u+\frac{k q}{p}, v+\frac{k}{p}\right) .
$$

In these coordinates, the $\mathbb{Z}_{p}^{q}$ orbit at the point $\left(z_{0}, w_{0}\right)=\varphi(\mathbb{Z} \times \mathbb{Z}) \in T_{r}$ is given by:

$$
\operatorname{Orbit}_{p, q}\left(z_{0}, w_{0}\right)=\left\{(m, n)+k\left(\frac{1}{p}, \frac{q}{p}\right): m, n, k \in \mathbb{Z}\right\} .
$$

Lemma 3.3 Given a sequence $\{L(p, q)\}_{p \in \mathbb{N}}$, there exist $b, m_{0}, n_{0} \in \mathbb{Z}$ and a subsequence $\left\{L\left(p_{l}, q_{l}\right)\right\}_{l \in \mathbb{N}}$ such that one of the following holds:

(1) For every $\left(z_{0}, w_{0}\right) \in T_{r}, \varphi_{r}\left(\right.$ Orbit $\left._{p_{l}, q_{l}}\left(z_{0}, w_{0}\right)\right)$ is becoming dense on $T_{r}$ as $l \rightarrow$ $\infty$.

(2) $\varphi_{r}\left(\right.$ Orbit $\left._{p_{l}, q_{l}}\left(z_{0}, w_{0}\right)\right)$ is contained in $b$ integral curves of $X(z, w)=\left(m_{0} \sqrt{-1} z, n_{0} \sqrt{-1} w\right) \in \mathcal{X}\left(\mathbb{S}^{3}\right)$.

Proof To prove the lemma it is enough to consider $\left(z_{0}, w_{0}\right) \in T_{\frac{\pi}{4}}$. If there is a subsequence for which the diameter of $T_{\frac{\pi}{4}} / \mathbb{Z}_{p}^{q}$ is going to zero as $p \rightarrow \infty$, then $\varphi_{\frac{\pi}{4}}\left(\operatorname{Orbit}_{p, q}\left(z_{0}, w_{0}\right)\right)$ is clearly becoming dense on $T_{\frac{\pi}{4}}$ and item 1 is proved.

Let us consider now the case where the diameter of $T_{\frac{\pi}{4}}$ in $L(p, q)$ is bounded away from zero. From the equivalence between extrinsic and intrinsic distance and by Lemma 4.2 we conclude that the Euclidean injectivity radius satisfies $\operatorname{inj}_{\left(z_{0}, w_{0}\right)} T_{\frac{\pi}{4}} / \mathbb{Z}_{p}=O\left(\frac{1}{p}\right)$. In particular, there exist $k_{p}, m_{p}, n_{p} \in \mathbb{Z}$ such that

$$
0<\left\|k_{p}\left(\frac{1}{p}, \frac{q}{p}\right)-\left(m_{p}, n_{p}\right)\right\|_{\mathbb{R}^{2}} \leq \frac{C}{p} .
$$

Therefore, there exists $\left(m_{0}, n_{0}\right) \in B_{C}(0) \cap \mathbb{Z} \times \mathbb{Z} \subset \mathbb{R}^{2}$ such that $\left(k_{p}-p m_{p}, k_{p} q-\right.$ $\left.n_{p} p\right)=\left(m_{0}, n_{0}\right)$ infinitely often and $\frac{\sqrt{m_{0}^{2}+n_{0}^{2}}}{2 p}$ is the Euclidean injectivity radius of 
$T_{\frac{\pi}{4}} / \mathbb{Z}_{p}$ at $\left(z_{0}, w_{0}\right)$ for this subsequence. Hence, the sub-orbit generated by the translation $(u, v) \rightarrow(u, v)+k_{p}\left(\frac{1}{p}, \frac{q}{p}\right)$ is contained in the line $\mathbb{Z} \times \mathbb{Z}+\left\{t\left(m_{0}, n_{0}\right): t \in \mathbb{R}\right\}$. It follows that the $\operatorname{Orbit}_{p, q}\left(z_{0}, w_{0}\right)$ is contained in a union of equidistant lines parallel to the one described above by homogeneity. Modulo $\mathbb{Z} \times \mathbb{Z}$ the number of such lines is finite, let's denote it by $b_{p}$. Modulo $\mathbb{Z} \times \mathbb{Z}$ there are $\frac{p}{b_{p}}$ points of Orbit ${ }_{p, q}\left(z_{0}, w_{0}\right)$ in each of these lines. Hence,

$$
\frac{p}{b_{p}}\left(\frac{k_{p}}{p}, \frac{k_{p} q}{p}\right)-\frac{p}{b_{p}}\left(m_{p}, n_{p}\right)=\frac{p}{b_{p}}\left(\frac{m_{0}}{p}, \frac{n_{0}}{p}\right) \in \mathbb{Z} \times \mathbb{Z} .
$$

Therefore, $b_{p}$ divides $m_{0}$ and is independent of $p$. In other words, $\varphi_{\frac{\pi}{4}}\left(\operatorname{Orbit}_{p, q}\left(z_{0}, w_{0}\right)\right)$ is contained in $b$ integral curves of $X(z, w)=\left(m_{0} \sqrt{-1} z, n_{0} \sqrt{-1} w\right) \in \mathcal{X}\left(\mathbb{S}^{3}\right)$.

\subsection{Cheeger-Gromov convergence}

A sequence of pointed Riemannian manifolds $\left(M_{i}, g_{i}, x_{i}\right)$ (Riemannian manifolds $\left(M_{i}, g_{i}\right)$ with points $\left.x_{i} \in M_{i}\right)$ is said to converge, in the sense of Cheeger-Gromov, to a pointed Riemannian manifold $(M, g, x)$ as $i \rightarrow \infty$ if the following two conditions hold true:

(1) There exists an exhaustion of $M$ by compact sets $\Omega_{i} \subset M: \bar{\Omega}_{i} \subset \Omega_{n+1}$ and $\bigcup_{i=1}^{\infty} \Omega_{i}=M$.

(2) There exists a family of smooth maps $\phi_{i}: \Omega_{i} \rightarrow M_{i}$ which are diffeomorphic onto their image such that $\phi_{i}(x)=x_{i}$ for all $i$ and $\phi_{i}^{*}\left(g_{i}\right) \rightarrow g$ in the $C^{\infty}$ topology.

In the next lemma we study the Cheeger-Gromov convergence for a sequence of lens spaces.

Recall that the rank of an orientable flat 3 -manifold $\mathbb{R}^{3} / G$ is the rank of the subgroup of translations in $G$.

Lemma 3.4 Let $\left(L(p, q), p^{2} g_{\mathbb{S}^{3}}, x_{p}\right)$ be a sequence of lens spaces. There exists a flat 3-manifold $\left(M, \delta, x_{\infty}\right)$ of rank at most one such that after passing to some subsequence $\left(L(p, q), p^{2} g_{\mathbb{S}^{3}}, x_{p}\right) \stackrel{C-G}{\longrightarrow}\left(M, \delta, x_{\infty}\right)$.

Proof Since $\left(L(p, q), p^{2} g_{\mathbb{S}^{3}}\right)$ has constant curvature converging to zero and $\operatorname{inj}\left(L(p, q), p^{2} g_{\mathbb{S}^{3}}\right) \geq \pi$, the Cheeger-Gromov Compactness Theorem [35, Theorem 7.1.3] implies that $\left(L(p, q), p^{2} g_{\mathbb{S}^{3}}, x_{p}\right) \stackrel{C-G}{\longrightarrow}\left(M, \delta, x_{\infty}\right)$, where $(M, \delta)$ is an orientable flat 3-manifold. Every non-compact flat 3-manifold is finitely covered by either $\mathbb{S}^{1} \times \mathbb{R}^{2}$ or $T^{2} \times \mathbb{R}$; hence, to show that rank of $M$ is one, it is enough to prove that that the volume growth of balls of large radius are at least quadratic.

Below we denote $T_{r} / \mathbb{Z}_{p}$ by $T_{r}$. Let $T_{r_{p}}$ be the Clifford torus through $x_{p}$ enclosing a region $\Omega_{r_{p}}$. Under the scaling by $\lambda_{p}=p^{2}$ we have that $\left|\Omega_{r_{p}}\right|=2 \pi^{2} p^{2} \sin ^{2}\left(r_{p}\right)$. If $\lim _{p \rightarrow \infty}\left|\Omega_{r_{p}}\right|<\infty$, then $\lim _{p \rightarrow \infty}\left|T_{r_{p}}\right|=2 \pi^{2} p \sin \left(2 r_{p}\right)<\infty$. Moreover, the second fundamental form $A_{r_{p}}$ of $T_{r_{p}}$ satisfies $\lim _{p \rightarrow \infty}\left|A_{r_{p}}\right|^{2}=\lim _{p \rightarrow \infty} \frac{1}{p^{2}}\left(\frac{\cos ^{2}\left(r_{p}\right)}{\sin ^{2}\left(r_{p}\right)}+\right.$ $\left.\frac{\sin ^{2}\left(r_{p}\right)}{\cos ^{2}\left(r_{p}\right)}\right)<\infty$. The critical fiber $T_{0} \subset \Omega_{r_{p}}$ is distant from $x_{p}$ by $O\left(\frac{1}{p}\right)$ since $\Omega_{r_{p}}$ 
is converging to a compact region in $M$. Instead of using base points $x_{p}$ we choose new base points $y_{p} \in T_{0}$; it follows that $\left(L(p, q), p^{2} g_{\mathbb{S}^{3}}, y_{p}\right) \rightarrow\left(N, \delta, y_{\infty}\right)$ and $\operatorname{rank}(N)=\operatorname{rank}(M)$. We claim that $\operatorname{rank}$ of $N$ is at most one:

$$
\left|B_{2 R}\left(y_{\infty}\right)\right|=\lim _{p \rightarrow \infty}\left|B_{2 R}^{p}\left(y_{p}\right)\right| \geq \lim _{p \rightarrow \infty}\left|\Omega_{\frac{R}{p}}\right|=\lim _{p \rightarrow \infty} 2 \pi^{2} p^{2} \sin ^{2}\left(\frac{R}{p}\right)=c R^{2} .
$$

Let's assume now that $\lim _{p \rightarrow \infty}\left|\Omega_{r_{p}}\right|=\infty$, consequently $\lim _{p \rightarrow \infty}\left|T_{r_{p}}\right|=\infty$ and $\lim _{p \rightarrow \infty}\left|A_{r_{p}}\right|^{2}=0$. Recall the function $r=r(x)$, the distance from the Clifford torus through $x$ to the critical fiber $T_{0}$ with respect to the round metric. The unit vector field $\partial r$ is orthogonal to $T_{r}$ for every $r$ and it is well defined on $L(p, q)-\left\{T_{0} \cup T_{\frac{\pi}{2}}\right\}$. Let $\gamma(r)$ be the geodesic whose velocity is $\partial r$ and such that $\gamma\left(r_{p}\right)=x_{p}$. Consider $K_{r_{p}, R}=\left\{x \in T_{r}: d_{L(p, q)}(x, \gamma(r)) \leq \frac{R}{p}\right.$ and $\left.\left|r-r_{p}\right| \leq \frac{R}{p}\right\}$. By the triangle inequality $K_{r_{p}, R} \subset B_{2 R}\left(x_{p}\right)$ under the metric $p^{2} g_{\mathbb{S}^{3}}$. Applying the coarea formula for $f(r)=$ pr, $|\nabla f|_{p^{2} g_{\mathbb{S}^{3}}}=1$, we obtain:

$$
\left|K_{r_{p}, R}\right|=\int_{r_{p}-\frac{R}{p}}^{r_{p}+\frac{R}{p}}\left|B_{R}(\gamma(u)) \cap T_{u}\right| p d u=\left|B_{R}\left(\gamma\left(u_{0}\right)\right) \cap T_{u_{0}}\right|_{p^{2} g_{\mathbb{S}^{3}}} R \geq c R^{2},
$$

where $u_{0} \in\left[r_{p}-\frac{R}{p}, r_{p}+\frac{R}{p}\right]$ is from the mean value theorem for integrals. The last inequality is justified as follows. Either the extrinsic diameter of $T_{u_{0}}$ is going to infinity and $T_{u_{0}}$ is converging with multiplicity to a flat surface or the extrinsic diameter of $T_{u_{0}}$ is bounded. The former implies that $\left|B_{R}\left(\gamma\left(u_{0}\right)\right) \cap T_{u_{0}}\right|_{p^{2} g_{\mathbb{S}^{3}}} \geq c R$. The latter implies that $B_{R}\left(\gamma\left(u_{0}\right)\right) \cap T_{u_{0}}=T_{u_{0}}$, which is a contradiction since $\left|T_{u_{0}}\right|_{p^{2} g_{\mathbb{S}^{3}}} \rightarrow \infty$. We conclude that $\operatorname{Vol}\left(B_{2 R}\right) \geq c R^{2}$ and rank of $M$ is at most one.

\section{Proof of Theorem 1.1}

Lemma 4.1 Let $\left(L(p, q), p^{2} g_{\mathbb{S}^{3}}, x_{p}\right) \rightarrow\left(M, \delta, x_{\infty}\right)$ be as in Lemma 3.4 and let $\Sigma_{p}$ be an isoperimetric surface in $L(p, q)$ such that $x_{p} \in \Sigma_{p}$ and $g\left(\Sigma_{p}\right) \geq 1$. There exists a constant $C>0$ such that $\left|A_{\Sigma_{p}}\right|_{p^{2} g_{\mathbb{S}^{3}}} \leq C$.

Proof Let $y_{p} \in \Sigma_{p} \subset L(p, q)$ be such that $\left|A_{p}\right|\left(y_{p}\right)=\max _{\Sigma_{p}}\left|A_{p}\right|^{2}$ and define $\lambda_{p}=$ $\max _{\Sigma_{p}}\left|A_{p}\right|\left(y_{p}\right)$. Arguing by contradiction, let's assume that $\frac{\lambda_{p}}{p} \rightarrow \infty$. In local coordinates around $y_{p}$ we consider the surface $\Sigma_{p}^{\prime}=\lambda_{p} \Sigma_{p}$ on the Euclidean ball $B_{\lambda_{p} \frac{\pi}{10 p}}(0)$ endowed with the rescaled metric $\lambda_{p}^{2} g_{\mathbb{S}^{3}}$. Therefore, $\left(B_{\lambda_{p} \frac{\pi}{10 p}}(0), \lambda_{p}^{2} g_{\mathbb{S}^{3}}, y_{p}\right)$ converges to $\left(\mathbb{R}^{3}, \delta, 0\right)$ as $p \rightarrow \infty$. The surface $\Sigma_{p}^{\prime}$ now has the property that $\max _{\Sigma_{p}^{\prime}}\left|A_{p}^{\prime}(0)\right|^{2}=1$.

By the strong compactness for a sequence of isoperimetric surfaces with bounded second fundamental form, see Corollary 6.4 in the Appendix, there exists a subsequence converging to a properly embedded surface $\Sigma^{\prime} \subset \mathbb{R}^{3}$, the convergence is in 
the sense of graphs and with multiplicity one. Moreover, $\Sigma^{\prime}$ is also stable, i.e.:

$$
I_{\Sigma^{\prime}}(f, f) \geq 0, \forall f \in C_{0}^{\infty}\left(\Sigma^{\prime}\right) \text { satisfying } \int_{\Sigma^{\prime}} f d_{\Sigma^{\prime}}=0
$$

If $\Sigma^{\prime}$ is compact, then it has to be a round sphere by Alexandrov's Theorem, which is a contradiction since strong convergence preserves topology. If $\Sigma^{\prime}$ is non-compact, then it has infinite area by the monotonicity formula: indeed, by Proposition 6.1 in the Appendix there exists a positive constant $C$ such that

$$
\frac{d}{d r}\left(\frac{e^{C r}\left|\Sigma^{\prime} \cap B_{r}(x)\right|}{r^{2}}\right) \geq 0
$$

In particular, $\left|\Sigma^{\prime} \cap B_{r}(x)\right| \geq \pi r^{2}$. As $\Sigma^{\prime}$ is properly embedded, it has infinite extrinsic diameter and the claim follows. Therefore, $\Sigma^{\prime}$ is totally geodesic by Da Silveira's Theorem 6.6 in the Appendix, which is a contradiction since $\max _{\Sigma^{\prime}}|A|=1$.

The following lemma gives a description of $I_{L(p, q)}$ for small volumes:

Lemma 4.2 For $p$ large enough there exist $v_{p}$ and $\varepsilon_{p}>0$ such that $I_{L(p, q)}$ is given by the profile of spheres on $\left(0, v_{p}\right]$ and by the profile of flat tori on $\left[v_{p}, v_{p}+\varepsilon_{p}\right)$. Moreover, if $\Sigma_{p}$ is an isoperimetric surface such that $I_{L(p, q)}\left(v_{p}\right)=\left|\Sigma_{p}\right|$, then $g\left(\Sigma_{p}\right)=0$ or 1 .

Proof For each $p$ we consider the first volume, $v_{p}$, for which there is transition on topology of isoperimetric surfaces from spheres to something else. If $v_{*}$ is the volume for which the profile of geodesic spheres intersect the profile of flat tori, then $v_{p} \leq v_{*}$. The value of $v_{*}$ is computed by solving the following system of equations:

$$
\frac{2 \pi^{2}}{p} \sin ^{2}(r)=2 \pi s-\pi \sin (2 s) \text { and } \frac{2 \pi^{2}}{p} \sin (2 r)=4 \pi \sin ^{2}(s) .
$$

The left hand side (right hand side) of the identities in (4.1) correspond to the enclosed volume and area of the Clifford torus $T_{r}$ (geodesic spheres $S_{S}$ of radius $s$ ), respectively. It follows that $s \leq \frac{\pi}{p}$; another way to see this is by recalling that the injectivity radius of $L(p, 1)$ is $\frac{\pi}{p}$ at every point. Therefore, $I_{L(p, q)}\left(v_{p}\right) \leq \frac{C}{p^{2}}$.

Let $\Sigma_{p}$ be an isoperimetric surface with genus $g\left(\Sigma_{p}\right) \geq 1$ and satisfying $I_{L(p, q)}\left(v_{p}\right)=\left|\Sigma_{p}\right|$. By Lemma 4.1 the sequence $\left\{\Sigma_{p}\right\}_{p \in \mathbb{N}}$ has bounded second fundamental form in $\left(L(p, q), p^{2} g_{\mathbb{S}^{3}}, x_{p}\right)$; thus, it strongly converges to a properly embedded surface $\Sigma$ of finite area in some orientable flat three manifold $(M, \delta)$ of rank at most one by Lemma 3.4. By the monotonicity formula, Proposition 6.1 in the Appendix, there exists $r>0$ such that $\operatorname{Area}\left(\Sigma \cap B_{r}(x)\right) \geq C r^{2}$ for every $x \in \Sigma$. Hence, the extrinsic diameter of $\Sigma$ is bounded. As $\Sigma$ is properly embedded, $\Sigma$ is closed. It follows that the pre-image $\widehat{\Sigma}$ of $\Sigma$ in $\mathbb{R}^{3}$ is contained in a solid cylinder. Hence, $\widehat{\Sigma}$ is an union of round spheres by Alexandrov's Theorem or is a surface of revolution about the axis of the cylinder by Theorem 6.5 in the Appendix. Therefore, 
$g(\Sigma)=0$ or 1 . From the strong compactness for isoperimetric surfaces, see Corollary 6.4 in the Appendix, we conclude that $g\left(\Sigma_{p}\right)=0$ or $1, v_{p}=v_{*}$ and the existence of the desired $\varepsilon_{p}>0$.

Claim Theorem 1.1 follows if we can show that the isoperimetric surfaces separating $L(p, q)$ in two regions of the same volume are tori.

Proof By the strong compactness for isoperimetric surfaces, Corollary 6.4, there exists $\widehat{v}$ such that if $\Sigma$ is an isoperimetric surface enclosing volume $v \in\left[\widehat{v}, \frac{\pi^{2}}{p}\right]$, then $\Sigma$ is a flat torus. It follows from Lemma 4.2 and for large $p$ that the isoperimetric profile $I_{L(p, q)}$ is given by the area of geodesic spheres for volumes in $\left(0, v_{p}\right]$ and by the area of flat tori for volumes in $\left[v_{p}, \varepsilon_{p}\right] \cup\left[\widehat{v}, \frac{\pi^{2}}{p}\right]$. In other words, if $f(v)$ is the function defined by $f(v)=\left|T_{r(v)}\right|$, where $T_{r(v)}$ is the Clifford torus enclosing a volume equal to $v$, then $I_{L(p, q)}(v)=f(v)$ on $\left[v_{p}, \varepsilon_{p}\right] \cup\left[\widehat{v}, \frac{\pi^{2}}{p}\right]$. It follows that $\phi(v)=f(v)-I_{L(p, q)}(v)$ has a local maximum point at $t_{*} \in\left(\varepsilon_{p}, \widehat{v}\right)$.

The claim will follow by exploring the weak differential equation for $I_{L(p, q)}$. From (2.7) we have

$$
f^{2}(v) f^{\prime \prime}(v)+\int_{T_{r(v)}}\left(2+\left|A_{r(v)}\right|^{2}\right) d_{T_{r(v)}}=0 .
$$

Let $\Sigma$ be an isoperimetric surface such that $I_{L(p, q)}\left(t_{*}\right)=|\Sigma|$. If $\Sigma_{v}$ is the unit normal variation of $\Sigma$ parametrized by the enclosed volume, then we define $h(v)=\operatorname{Area}\left(\Sigma_{v}\right)$. Since $h \geq I_{L(p, q)}$, we have that $\phi_{1}=f-h$ has also a local maximum point at $t_{*}$. Hence, $\phi_{1}^{\prime}\left(t_{*}\right)=0$, i.e., $H_{r}=H$, and $\phi_{1}^{\prime \prime}\left(t_{*}\right) \leq 0$. Applying Eq. (2.7) for $h$ together with the Gauss equation and the Gauss-Bonnet theorem we obtain:

$$
\begin{aligned}
\phi_{1}^{\prime \prime}\left(t_{*}\right) \leq 0 & \Rightarrow \frac{1}{h^{2}} \int_{\Sigma}\left(2+|A|^{2}\right) d_{\Sigma} \leq \frac{1}{f^{2}} \int_{T_{r\left(t_{*}\right)}}\left(2+\left|A_{r(v)}\right|^{2}\right) d_{T_{r\left(t_{*}\right)}} \\
& \Rightarrow 4\left(1+H^{2}\right) h+8 \pi(g-1) \leq\left(4\left(1+H_{r}^{2}\right) f\right) \frac{h^{2}}{f^{2}} \\
& \Rightarrow 1+H^{2}+\frac{2 \pi(g-1)}{h} \leq\left(1+H_{r}^{2}\right) \frac{h}{f} \leq 1+H^{2} .
\end{aligned}
$$

Therefore, $g(\Sigma)=1$ and $I_{L(p, q)}=f$ in $\left[v_{p}, \frac{\pi^{2}}{p}\right]$.

Proof of Theorem 1.1 Arguing by contradiction, let us assume that there exists an infinite sequence of lens spaces $L(p, q)$ containing an isoperimetric surface $\Sigma_{p}$ of genus $g \geq 2$ for each $p$. By the claim above, we may assume that $\Sigma_{p}$ divides $L(p, q)$ in two regions of equal volume.

We consider pointed manifolds $\left(L(p, q), p^{2} g_{\mathbb{S}^{3}}, x_{p}\right)$ with base points $x_{p}$ belonging to $\Sigma_{p}$. By the Cheeger-Gromov compactness theorem, $\left(L(p, q), p^{2} g_{\mathbb{S}^{3}}, x_{p}\right) \stackrel{C-G}{\longrightarrow}$ $\left(M, \delta, x_{\infty}\right)$, where $(M, \delta)$ is a flat three manifold. The inclusion of $\Sigma_{p}$ into $M$ through the diffeomorphism $\phi_{p}$ is still denoted by $\Sigma_{p}$. 
By Lemma 3.3, the proof of Theorem 1.1 reduces to investigating the Cases I and II below.

Case I: There is a subsequence whose $\mathbb{Z}_{p}^{q}$ orbit of a point is contained in a finite number (independent of $p$ ) integral curves of a vector field $X(z, w)=$ $\left(m_{0} \sqrt{-1} z, n_{0} \sqrt{-1} w\right) \in \mathcal{X}\left(\mathbb{S}^{3}\right)$.

We claim that the injectivity radius of $L(p, q)$ at every point is $O\left(\frac{1}{p}\right)$. Indeed, let Orbit $_{p, q}\left(z_{0}, w_{0}\right)$ be the orbit of $\left(z_{0}, w_{0}\right) \in \mathbb{S}^{3}$ with respect to $\mathbb{Z}_{p}^{q}$. As before, $\frac{p}{b}$ points of Orbit $_{p, q}\left(z_{0}, w_{0}\right)$ lie on the curve $\beta(t)=\psi\left(z_{0}, w_{0}, t\right)$. Here, $\psi$ is the one parameter family of diffeomorphisms associated to $X$ :

$$
\psi: \mathbb{S}^{3} \times \mathbb{R} \rightarrow \mathbb{S}^{3}: \psi(z, w, t)=\left(e^{m_{0} i t} z, e^{n_{0} i t} w\right) .
$$

When ordered according the orientation of $\beta(t)$, those points determine a piecewise closed geodesic $\gamma_{p}(t)$ with $\gamma_{p}(0)=\left(z_{0}, w_{0}\right)$. As $p \rightarrow \infty, \gamma_{p}(t)$ converges to $\beta(t)$. The claim now follows from:

$$
\lim _{p \rightarrow \infty} \frac{p}{b} \operatorname{inj}_{\left(z_{0}, w_{0}\right)} L(p, q) \leq \lim _{p \rightarrow \infty} \mathcal{H}^{1}\left(\gamma_{p}\right)=2 \pi \sqrt{m_{0}^{2}\left|z_{0}\right|^{2}+n_{0}^{2}\left|w_{0}\right|^{2}} .
$$

After extracting a subsequence, Lemma 3.4, implies that

$$
\left(L(p, q), p^{2} \cdot g_{\mathbb{S}^{3}}, x_{p}\right) \stackrel{C-G}{\longrightarrow}\left(M, \delta, x_{\infty}\right),
$$

where $\left(M, \delta, x_{\infty}\right)$ is a flat three manifold of rank at most one. By (4.2) $M=\mathbb{R}^{3} / S_{\theta}$, where $\theta \in \mathbb{Q}$.

The curves $t \rightarrow \beta(t)=\psi(x, t)$ represent integral curves of $X$ through $x \in L(p, q)$; they have bounded geodesic curvature and $\mathcal{H}^{1}(\beta)=O\left(\frac{1}{p}\right)$. Hence, the integral curves of $X$ converge to closed geodesics in $M$ under (4.3). As sets, they coincide with the standard vertical fibers of $M$. By the Poincaré-Hopf index theorem, the vector field $\frac{X^{\top}}{|X|} \in \mathcal{X}\left(\Sigma_{p}\right)$ has a zero since $g\left(\Sigma_{p}\right) \geq 2$. Hence, we can choose the base points $x_{p}$ to satisfy $g_{\mathbb{S}^{3}}\left(\frac{X}{|X|}\left(x_{p}\right), N\left(x_{p}\right)\right)= \pm 1$, here $N$ is the unit normal vector of $\Sigma_{p}$.

Lemma 4.3 There exists a properly embedded surface $\Sigma_{\infty} \subset M$ such that $\left(\Sigma_{p}, x_{p}\right) \rightarrow$ $\left(\Sigma_{\infty}, x_{\infty}\right)$ with multiplicity one. Moreover, $\Sigma_{\infty}$ is totally geodesic and perpendicular to the standard fibers of $M$.

Proof By Lemma 4.1 the sequence of isoperimetric surfaces $\left(\Sigma_{p}, x_{p}\right) \subset(L(p, q)$, $p^{2} g_{\mathbb{S}^{3}}, x_{p}$ ) has uniformly bounded second fundamental form. Applying the strong compactness theorem for isoperimetric surfaces, Corollary 6.4, we conclude that $\Sigma_{p}$ converges smoothly and with multiplicity one to a properly embedded stable cmc surface $\Sigma_{\infty} \subset \mathbb{S}^{1} \times \mathbb{R}^{2}$. If lim inf $\operatorname{li\infty }_{p \rightarrow \infty}\left|\Sigma_{p}\right|_{p^{2} g_{\mathbb{S}^{3}}}<\infty$, then the monotonicity formula, Proposition 6.1, implies that the extrinsic diameter of $\Sigma_{p}$ and $\Sigma_{\infty}$ are bounded. This is impossible since the sequence $\Sigma_{p}$ separates $L(p, q)$ in two regions of the same volume that goes to infinity as $p \rightarrow \infty$. Therefore, $\Sigma_{\infty}$ is a complete properly embedded stable cmc surface in $M$ with infinite area. Applying Da Silveira's Theorem 6.6 once more, we obtain that $\Sigma_{\infty}$ is totally geodesic. As $g_{\mathbb{S}^{3}}\left(\frac{X}{|X|}\left(x_{p}\right), N\left(x_{p}\right)\right)= \pm 1$, we conclude that $\Sigma_{\infty}$ is orthogonal to the standard fibers of $M$. 
R.

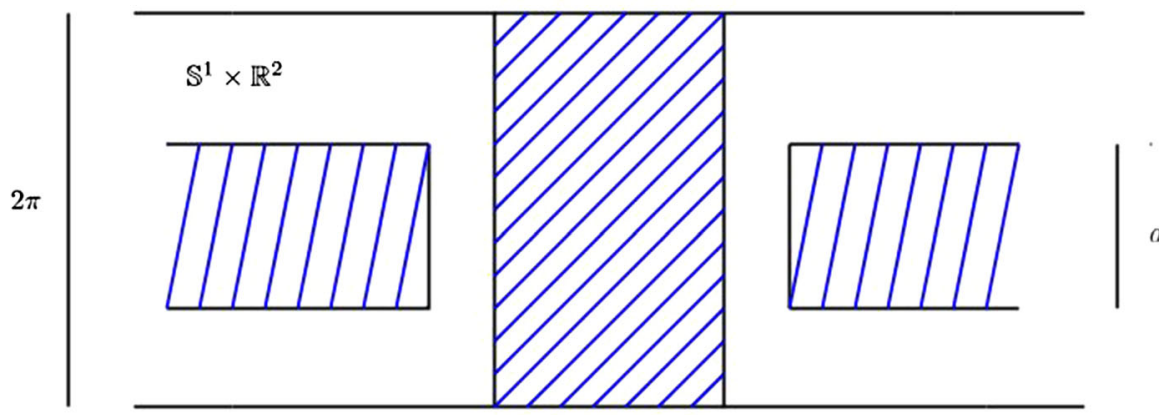

$r$

Fig. 1 Compact support deformation of $\Sigma_{\infty}$

We claim that $\Sigma_{\infty}$ separates $M$. If it does not separate, then there exists a loop $\gamma$ intersecting $\Sigma_{\infty}$ at a single point. As $\Sigma_{p} \rightarrow \Sigma_{\infty}$ with multiplicity one, the same conclusion holds for $\Sigma_{p}$, which contradicts the fact that $\Sigma_{p}$ separates $L(p, q)$. Therefore, there is $k \geq 1$ such that $\Sigma_{\infty}=\partial \Omega_{\infty}=\bigcup_{i=1}^{2 k} \sigma_{i}$, where $\sigma_{i}$ is a flat plane for each $i$.

Claim This configuration cannot be a limit of isoperimetric surfaces.

We regard $M$ as a slab in $\mathbb{R}^{3}$ with height $2 \pi$. Now, we construct a deformation of $\Sigma_{\infty}$ which decreases its area as follows. First, we cut off the $k$ solid cylinders obtained from the intersection of $\Omega_{\infty}$ with a vertical solid torus of radius $R$. To balance the enclosed volume we add a vertical solid torus of radius $r$, see Fig. 1 for the case $k=1$. If $a_{i}$ is the distance between $\sigma_{2 i}$ and $\sigma_{2 i-1}$, then the radius $r$ is given by:

$$
\sum_{i=1}^{k} \pi R^{2} a_{i}=\pi r^{2} 2 \pi \Rightarrow r=R \sqrt{\frac{\sum_{i} a_{i}}{2 \pi}}
$$

The boundary of this new region is denoted by $\widetilde{\Sigma}_{\infty}$ and

$$
\operatorname{Area}\left(\widetilde{\Sigma}_{\infty} \cap K\right)=\operatorname{Area}\left(\Sigma_{\infty} \cap K\right)-2 k \pi R^{2}+2 \pi R \cdot \sum_{i} a_{i}+2 \pi r \cdot 2 \pi
$$

If $R$ is large enough, then $\widetilde{\Sigma}_{\infty}$ has less area than $\Sigma_{\infty}$. This is impossible since the strong multiplicity one convergence allow us to carry out this deformation of $\Sigma_{\infty}$ to $\Sigma_{p}$ which contradicts the fact that $\Sigma_{p}$ is an isoperimetric surface.

Case II: There is a subsequence $p \rightarrow \infty$ where the $\mathbb{Z}_{p}^{q}$ orbit of a point is becoming dense on the Clifford torus containing such point.

We follow a different strategy in this case. Instead of working with the limit manifold $M$, we use geometric measure theory methods to analyze the pre-image sequence $\left\{\Sigma_{p}^{\prime}\right\}_{p \in \mathbb{N}} \subset \mathbb{S}^{3}$. 
It is proved in Ref. [30] that if $\Sigma=\partial \Omega$ has positive mean curvature, then

$$
3|\Omega| \leq \int_{\Sigma} \frac{1}{H} d_{\Sigma} .
$$

Applying this formula to the sequence of isoperimetric surfaces, we conclude that the mean curvature of $\Sigma_{p}$ satisfies $H_{p} \leq \frac{\left|\Sigma_{p}\right|}{3\left|\Omega_{p}\right|} \leq \frac{2}{3}$ since $\left|\Sigma_{p}\right| \leq \frac{2 \pi^{2}}{p}$ and $\left|\Omega_{p}\right|=\frac{\pi^{2}}{p}$. We used that $\Sigma_{p}$ separates $L(p, q)$ into regions of the same volume.

As $\left\{\Sigma_{p}\right\}_{p \in \mathbb{S}^{3}}$ has area and mean curvature bounded, we apply Allard's compactness theorem, Theorem 42.7 and Remark 42.8 in Ref. [34], to obtain an integral varifold $0 \neq \mathcal{V}^{2} \subset \mathbb{S}^{3}$ that, up to subsequence, is $\mathcal{V}^{2}=\lim _{p \rightarrow \infty} \Sigma_{p}^{\prime}$. Recall that $\mathbb{S}^{1} \times \mathbb{S}^{1}$ acts on $\mathbb{S}^{3}$ via $(z, w) \rightarrow\left(\alpha_{1}, \alpha_{2}\right)(z, w):=\left(\alpha_{1} z, \alpha_{2} w\right)$. We claim that $\mathcal{V}^{2}$ is $\mathbb{S}^{1} \times \mathbb{S}^{1}$ invariant: indeed, if $(z, w) \in \operatorname{supp}\left(\mathcal{V}^{2}\right)$ and $\left(\alpha_{1}, \alpha_{2}\right) \in \mathbb{S}^{1} \times \mathbb{S}^{1}$, then for each $p$ there is $l_{p} \in \mathbb{Z}$ such that $\lim _{p \rightarrow \infty}\left(e^{\frac{2 \pi i l_{p} q}{p}} z, e^{\frac{2 \pi i l_{p}}{p}} w\right)=\left(\alpha_{1} z, \alpha_{2} w\right)$. On the other hand, as $(z, w) \in \mathcal{V}^{2}$, there is $\left(z_{p}, w_{p}\right) \in \Sigma_{p}^{\prime}$ such that $\left(e^{\frac{2 \pi i l_{p} q}{p}} z_{p}, e^{\frac{2 \pi i l_{p}}{p}} w_{p}\right) \in \Sigma_{p}^{\prime}$ and $\lim _{p \rightarrow \infty}\left(z_{p}, w_{p}\right)=(z, w)$. It follows that $\lim _{p \rightarrow \infty}\left(e^{\frac{2 \pi i l_{p} q}{p}} z_{p}, e^{\frac{2 \pi i l_{p}}{p}} w_{p}\right)=$ $\left(\alpha_{1} z, \alpha_{2} w\right)$ and $\left(\alpha_{1}, \alpha_{2}\right)(z, w) \in \operatorname{supp}\left(\mathcal{V}^{2}\right)$ by the monotonicity formula Proposition 6.1. In particular, supp $\mathcal{V}^{2}=\bigcup_{j=1}^{k} T_{r_{j}}$ where $T_{r_{j}}$ is a Clifford torus. The monotonicity formula implies that the convergence $\Sigma_{p}^{\prime} \rightarrow \mathcal{V}^{2}$ is also in Hausdorff distance; hence, we have that $\Sigma_{p}^{\prime}=\cup_{j=1}^{k} \Sigma_{p}^{\prime j}$ and supp $\left(\lim _{p \rightarrow \infty} \Sigma_{p}^{\prime j}\right)=T_{r_{j}}$. Since $\Sigma_{p}^{\prime}$ is $\mathbb{Z}_{p}^{q}$ invariant there exists $\theta_{p}^{j_{1} j_{2}} \in \mathbb{Z}_{p}^{q} \subset \mathbb{S}^{1} \times \mathbb{S}^{1}$ for which $\theta_{p}^{j_{1} j_{2}}\left(\Sigma_{i}^{\prime j_{2}}\right)=\Sigma_{p}^{\prime j_{1}}$. By taking the limit we obtain $\left(\alpha_{1}, \alpha_{2}\right)\left(T_{r_{j_{2}}}\right)=T_{r_{j_{1}}}$ for some $\left(\alpha_{1}, \alpha_{2}\right) \in \mathbb{S}^{1} \times \mathbb{S}^{1}$. As this is impossible, we conclude that $k=1$ and all $\Sigma_{p}^{\prime}$ are connected for $p$ large.

Now we consider $\left\{\Sigma_{p}^{\prime}\right\}_{p=1} \subset \mathbf{I}_{2}\left(\mathbb{S}^{3}, \mathbb{Z}\right)$, the space of 2-dimensional integral currents on $\mathbb{S}^{3}$. Each $\Sigma_{p}^{\prime}=\partial \Omega_{p}^{\prime}$ and $\Omega_{p}^{\prime} \in \mathbf{I}_{3}\left(\mathbb{S}^{3}, \mathbb{Z}\right)$. As $\Omega_{p}^{\prime}$ is a region of finite perimeter ( $\mathcal{X}_{\Omega_{p}^{\prime}}$ is BV function with uniform bounded variation), then $\Omega_{i}^{\prime} \rightarrow \Omega^{\prime}$ and $\Sigma_{p}^{\prime} \rightarrow \partial \Omega^{\prime}$ as currents, $\Omega^{\prime}$ is an open set of finite perimeter, see Theorem 6.3 and proof of Theorem 37.2 in Ref. [34]. Since $\left|\Omega_{p}\right|=\pi^{2}$ we conclude that $\left|\Omega^{\prime}\right|=\pi^{2}$. Applying the Constancy theorem, Theorem 26.27 in Ref. [34], we conclude that $\Omega^{\prime}$ is the handlebody bounded by the Clifford torus $T_{r_{1}}$, and consequently $r_{1}=\frac{\pi}{4}$.

We proved that $\mathcal{V}^{2}=m T_{\frac{\pi}{4}}$ for some positive integer $m \in \mathbb{N}$. Since $\Sigma_{p}$ is isoperimetric, it follows that $m=1$. Indeed,

$$
m\left|T_{\frac{\pi}{4}}\right|=\left|\mathcal{V}^{2}\right|=\lim _{p \rightarrow \infty}\left|\Sigma_{p}^{\prime}\right| \leq\left|T_{\frac{\pi}{4}}\right|
$$

As $T_{\frac{\pi}{4}}$ is smooth, we have for $r>0$ sufficiently small that the density $\theta\left(T_{\frac{\pi}{4}}, r, x\right) \leq$ $1+\frac{\epsilon}{2}$, where $\epsilon>0$ is from Theorem 6.2 in the Appendix. On the other hand, as $\Sigma_{p}^{\prime}$ is converging to $T_{\frac{\pi}{4}}$ with multiplicity one, then $\theta\left(\Sigma_{p}^{\prime}, x, r\right) \leq 1+\epsilon$ for $p$ large enough. Now we invoke the smooth version of Allard's Regularity Theorem, Theorem 6.2, to concluded that the convergence $\Sigma_{p}^{\prime} \rightarrow T_{\frac{\pi}{4}}$ is strong, i.e., graphical with multiplicity one. As strong convergence preserves topology, we conclude that $g\left(\Sigma_{p}\right)=1$. This completes the proof of Theorem 1.1. 


\section{Berger spheres}

Let $g_{0}$ be the round metric on $\mathbb{S}^{3}$ and $J$ the vector field on $\mathbb{S}^{3}$ defined as $J(z, w)=$ $(\sqrt{-1} z, \sqrt{-1} w)$. Recall that $J$ is tangent to the fibers of the Hopf fibration $h: \mathbb{S}^{3} \rightarrow$ $\mathbb{S}^{2}\left(\frac{1}{2}\right)$.

The Berger metrics are Riemannian metrics $g_{\varepsilon}$ on $\mathbb{S}^{3}$ defined as:

$$
g_{\varepsilon}(X, Y)=g_{0}(X, Y)+\left(\varepsilon^{2}-1\right) g_{0}(X, J) g_{0}(Y, J), \quad \varepsilon>0
$$

The Riemannian manifolds $\left(\mathbb{S}^{3}, g_{\varepsilon}\right)$ are called the Berger spheres, they are denoted by $\mathbb{S}_{\varepsilon}^{3}$. Geometrically, the metric $g_{\varepsilon}$ shrinks the Hopf fibers to have length $2 \pi \varepsilon$.

The Berger metrics are also homogeneous and their group of isometries has dimension four. It follows from the work of Abresch and Rosenberg [1] that every constant mean curvature surface in $\mathbb{S}_{\varepsilon}^{3}$ admits a holomorphic quadratic differential. In particular, every cmc sphere in $\mathbb{S}_{\varepsilon}^{3}$ is rotationally invariant.

A precise study of closed orientable surfaces with constant mean curvature on the Berger spheres is given in Ref. [36]. It is proved there the existence of $\varepsilon_{1}>0$ with the following property: if $\varepsilon \in\left[\varepsilon_{1}, 1\right]$, then every stable constant mean curvature surface in $\mathbb{S}_{\varepsilon}^{3}$ has genus zero or one. Moreover, if $\varepsilon^{2} \in\left[\frac{1}{3}, 1\right]$, then these stable cmc surfaces are totally umbilical spheres or the minimal Clifford torus, the latter only occurring when $\varepsilon^{2}=\frac{1}{3}$. In particular, rotationally invariant spheres are the only solutions of the isoperimetric problem in $\mathbb{S}_{\varepsilon}^{3}$ for $\varepsilon^{2} \in\left[\frac{1}{3}, 1\right]$.

Theorem 5.1 There exists $\varepsilon_{0}>0$ such that for every $\varepsilon<\varepsilon_{0}$ the isoperimetric surfaces in the Berger spheres $\mathbb{S}_{\varepsilon}^{3}$ are either rotationally invariant spheres or tori.

Remark 5.2 It follows from the works [21,25] that the minimal Clifford torus $\mathbb{S}_{\varepsilon}^{3}$ is isoperimetric for every $\varepsilon \leq \frac{1}{2}$.

Proof of Theorem 1.2 Arguing by contradiction, let us assume the existence of a sequence $\varepsilon \rightarrow 0$ such that for every $\varepsilon$ there exists an isoperimetric surface $\Sigma_{\varepsilon}$ in $\mathbb{S}_{\varepsilon}^{3}$ with $g\left(\Sigma_{\varepsilon}\right) \geq 2$.

We rescale the metric $g_{\varepsilon}$ of $\mathbb{S}_{\varepsilon}^{3}$ by the factor $\lambda_{\varepsilon}=\frac{1}{\varepsilon^{2}}$. The Hopf fibers have constant length equal to $2 \pi$ under the new metric $\lambda_{\varepsilon} g_{\varepsilon}$. It follows that the injective radius of $\mathbb{S}_{\varepsilon}^{3}$ at a point $p$ is equal to $i n j_{p} \mathbb{S}_{\varepsilon}^{3}=\pi$ for every $p \in \mathbb{S}_{\varepsilon}^{3}$.

Since $h$ is a local trivial fibration, we have that for each $p_{\varepsilon} \in \mathbb{S}_{\varepsilon}^{3}$ there exist a neighbourhood $V$ of $h\left(p_{\varepsilon}\right)$ and a diffeomorphism $\phi_{\varepsilon}: V \times \mathbb{S}^{1} \rightarrow h^{-1}(V)$ such that $h \circ \phi_{\varepsilon}=\pi_{1}$, where $\pi_{1}: V \times \mathbb{S}^{1} \rightarrow V$ given by $\pi(x, y)=x$. Moreover, $\phi_{\varepsilon}^{*}\left(\frac{1}{\varepsilon^{2}} g_{\varepsilon}\right) \rightarrow \delta$ in the $C^{\infty}$ topology. Therefore, in the sense of Cheeger-Gromov we have:

$$
\left(\mathbb{S}_{\varepsilon}^{3}, \frac{1}{\varepsilon^{2}} g_{\varepsilon}, p_{\varepsilon}\right) \rightarrow\left(\mathbb{S}^{1} \times \mathbb{R}^{2}, \delta, 0\right) .
$$

We pick the points $p_{\varepsilon} \in \Sigma_{\varepsilon}$ with the property that $g_{\varepsilon}\left(J, N_{\varepsilon}\right)\left(p_{\varepsilon}\right)= \pm \varepsilon$, this means $J$ and $N_{\varepsilon}$ are parallel at $p_{\varepsilon}$. These points exist by the Poincaré-Hopf index theorem. By Lemma 4.1 the inclusion of $\Sigma_{\varepsilon}$ in $\left(\mathbb{S}^{1} \times \mathbb{R}^{2}, \phi_{\varepsilon}^{*}\left(\varepsilon^{-2} g_{\varepsilon}\right)\right)$ has the following 
property:

$$
\text { There exists } C>0 \text { such that } \sup _{\Sigma_{\varepsilon}}\left|A_{\varepsilon}\right| \leq C \text { for every } \varepsilon \text {. }
$$

By the strong compactness theorem for isoperimetric surfaces, Corollary 6.4 in the Appendix, we can extract a subsequence, $\left\{\Sigma_{\varepsilon_{n}}\right\}$, which converges with multiplicity one to a properly embedded surface $\Sigma_{\infty} \subset\left(\mathbb{S}^{1} \times \mathbb{R}^{2}, \delta\right)$.

If $\operatorname{Area}\left(\Sigma_{\infty}\right)<\infty$, then the monotonicity formula, Proposition 6.1, implies that $\Sigma_{\infty}$ is compact. We apply Theorem 6.5 to conclude that $\Sigma_{\infty}$ is either a round sphere or torus. This is impossible since we have strong convergence and $g\left(\Sigma_{\varepsilon}\right)=2$ or 3 . Therefore, $\Sigma_{\infty}$ is a complete non-compact surface with infinite area. Moreover, $\Sigma_{\infty}$ is also a stable CMC surface in $\mathbb{S}^{1} \times \mathbb{R}^{2}$ :

$$
I_{\Sigma_{\infty}}(f, f) \geq 0, \quad \forall f \in C_{0}^{\infty}\left(\Sigma_{\infty}\right) \text { such that } \int_{\Sigma_{\infty}} f d_{\Sigma_{\infty}}=0 .
$$

It follows from Theorem 6.6 that $\Sigma_{\infty}$ is totally geodesic. By the choice of $p_{\varepsilon}$ we conclude that $\Sigma_{\infty}$ is orthogonal to the $\mathbb{S}^{1}$ fibers of $\mathbb{S}^{1} \times \mathbb{R}^{2}$. Since $\Sigma_{\infty}$ separates $\mathbb{S}^{1} \times \mathbb{R}^{2}$, we also conclude that $\Sigma_{\infty}$ is an union of at least two totally geodesic planes. As shown in the proof of Theorem 1.1, this configuration cannot be a limit of isoperimetric surfaces.

Acknowledgements I am grateful to my advisor André Neves for his constant encouragement and support during the course of this work. I also thank Jason Lotay for many helpful comments on an earlier version of this manuscript. This work was supported by the Engineering and Physical Sciences Research Council [EP/L015234/1]. The EPSRC Centre for Doctoral Training in Geometry and Number Theory (The London School of Geometry and Number Theory), University College London.

Open Access This article is distributed under the terms of the Creative Commons Attribution 4.0 International License (http://creativecommons.org/licenses/by/4.0/), which permits unrestricted use, distribution, and reproduction in any medium, provided you give appropriate credit to the original author(s) and the source, provide a link to the Creative Commons license, and indicate if changes were made.

\section{Appendix}

In this Appendix we collect some background results for surfaces with constant mean curvature in 3-manifolds.

Proposition 6.1 Let $M^{3}$ be a 3-manifold with bounded curvature, $\left|K_{M}\right| \leq k$, and with positive lower bound on the injectivity radius $\operatorname{inj}(M) \geq i_{0}$. If $\Sigma \subset M^{3}$ is a smooth surface with mean curvature which satisfies $|H| \leq H_{0}$, then there exists a positive constant $C=C\left(H_{0}, k\right)$ such that

$$
\frac{d}{d r}\left(\frac{e^{C r} \operatorname{Area}\left(\Sigma \cap B_{r}(p)\right)}{r^{2}}\right) \geq 0,
$$

for every $p \in \Sigma$ and $r \leq \min \left\{i_{0}, \frac{1}{\sqrt{k}}\right\}$. 
Proof See Chapter 7 in Ref. [10].

Let $\Sigma$ be a CMC surface in a closed manifold $M^{3}$. The density of $\Sigma$ at $x$ is given by

$$
\theta(\Sigma, x, r)=\frac{\operatorname{Area}\left(\Sigma \cap B_{r}(x)\right)}{\pi r^{2}}
$$

Theorem 6.2 (Allard's Regularity Theorem) Let $M^{3}$ be a closed manifold and $\rho>0$. There exist $\epsilon=\epsilon(M, \rho)>0$ and $C=C(M, \rho)$ with the following property: if $\Sigma \subset M$ is a smooth embedded CMC surface satisfying

$$
\theta(\Sigma, x, r) \leq 1+\epsilon
$$

for every $x \in M$ and $r<\rho$, then its second fundamental form is uniformly bounded, i.e., $\left|A_{\Sigma}\right| \leq C$.

Proof See Theorem 1.1 in Ref. [37].

Let $\left\{\Sigma_{n}\right\}_{n \in \mathbb{N}}$ be a sequence of surfaces in a manifold $M$. We say that $\Sigma_{n}$ converge to $\Sigma$ in the sense of graphs if near any point $p \in \Sigma$ and for large $n$ the surface $\Sigma_{n}$ is locally a graph over an open set of $T_{p} \Sigma$ and these graphs converge smoothly to the graph of $\Sigma$. In addition, we say that $\left\{\Sigma_{n}\right\}$ satisfy local area bounds if there exist $r>0$ and $C>0$ such that $\left|\Sigma_{n} \cap B_{r}(x)\right| \leq C$ for every $x \in M$.

A hypersurface $\Sigma$ is said to be weakly embedded if it admits only tangential self intersections.

Proposition 6.3 Let $\left\{\Sigma_{n}\right\} \subset\left(M, g_{n}\right)$ be a sequence of embedded surfaces with constant mean curvature satisfying local area bounds and such that $\sup _{\Sigma_{n}}\left|A_{n}\right| \leq C$. Let's assume that $g_{n}$ converges to a metric $\delta$ in the $C^{\infty}$ topology. If $\left\{\Sigma_{n}\right\}_{n=1}$ has an accumulation point, then we can extract a subsequence that converges to a properly weakly embedded cmc surface $\Sigma$ in $(M, \delta)$.

Sketch of the Proof Let's first recall the constant mean curvature equation for graphs. If $\Sigma^{\prime}$ is a surface with constant mean curvature $H$ in $(M, g)$, then $\Sigma^{\prime}$ can be written locally as a graph over a neighbourhood $U_{p} \subset T_{p} \Sigma^{\prime}$ :

$$
\Sigma^{\prime}=\operatorname{Graph}(u)=\left\{\left(x_{1}, x_{2}, u\left(x_{1}, x_{2}\right): x_{1}, x_{2} \in U_{p}\right)\right\} .
$$

In coordinates $g_{i j}:=g\left(e_{i}, e_{j}\right)$ where $\left\{e_{1}, e_{2}, e_{3}\right\}$ is the coordinate base associated to $\left(x_{1}, x_{2}, x_{3}\right)$. Let $\left\{E_{1}, E_{2}\right\}$ be the coordinate base for $\Sigma_{n}$, i.e., $E_{i}=e_{i}+u_{x_{i}} e_{3}=T_{i}^{l} e_{l}$. The induced metric $h$ is expressed by $h_{i j}=h\left(E_{i}, E_{j}\right)$. A simple computation gives:

$$
g\left(N, e_{i}\right)=\frac{-u_{x_{i}}}{\sqrt{1+g^{i j} u_{x_{i}} u_{x_{j}}}} \text { and } g\left(N, e_{3}\right)=\frac{1}{\sqrt{1+g^{i j} u_{x_{i}} u_{x_{j}}}} .
$$

We also have

$$
\nabla_{E_{i}} E_{j}=T_{i}^{l} \nabla_{e_{l}} T_{j}^{m} e_{m}=T_{i}^{l} T_{j}^{m} \nabla_{e_{l}} e_{m}+E_{i}\left(T_{j}^{m}\right) e_{m}
$$




$$
=T_{i}^{l} T_{j}^{m} \Gamma_{m l}^{k} e_{k}+u_{x_{i} x_{j}} e_{3},
$$

where $\Gamma_{m l}^{k}$ are the Christoffel symbols of $g$. Therefore,

$$
g\left(N, \nabla_{E_{i}} E_{j}\right)=-\frac{\sum_{k=1}^{2} T_{i}^{l} T_{j}^{m} \Gamma_{m l}^{k} u_{x_{k}}}{\sqrt{1+g^{i j} u_{x_{i}} u_{x_{j}}}}+\frac{\left(u_{x_{i} x_{j}}+T_{i}^{l} T_{j}^{m} \Gamma_{m l}^{3}\right)}{\sqrt{1+g^{i j} u_{x_{i}} u_{x_{j}}}} .
$$

Finally, the mean curvature equation is written as:

$$
H \sqrt{1+g^{i j} u_{x_{i}} u_{x_{j}}}=h^{i j} u_{x_{i} x_{j}}+h^{i j} T_{i}^{l} T_{j}^{m} \Gamma_{m l}^{3}-\sum_{k=1}^{2} h^{i j} T_{i}^{l} T_{j}^{m} u_{x_{k}} \Gamma_{m l}^{k}
$$

Since $h_{i j}=T_{i}^{l} T_{j}^{m} g_{m l}$ and $T_{i}^{3}=u_{x_{i}}$, then $\left|h^{i j}\right| \leq C_{1}\left(g^{i j}, u_{x_{i}}, u_{x_{j}}\right)$. The Eq. (6.2) is uniformly elliptic as long as $|\nabla u|,\left|\nabla^{2} u\right|<\widetilde{C}$.

Let $p \in M$ be an accumulation point for the sequence $\left\{\Sigma_{n}\right\}$. By the upper bound on the second fundamental form, $\sup _{\Sigma_{n}}\left|A_{n}\right|<C$, there exists $r_{0}=r_{0}(C)$ such that for every $q \in B_{r_{0}}(p) \cap \Sigma_{n}$ we have that $\Sigma_{n} \cap B_{r_{0}}(q)$ is locally a graph $u_{n}$ over a neighbourhood $U_{q} \subset T_{q} \Sigma_{n}$. Moreover, there exists $C_{2}=C_{2}(C)>0$ for which $\max \left\{\nabla u_{n}, \nabla^{2} u_{n}\right\} \leq C_{2}$. As $\left|g_{n}-\delta\right|_{C^{2, \alpha}} \rightarrow 0$, the Schauder estimates for solutions of elliptic equations, see [13], imply that $u_{n}$ have $C^{2, \alpha}$ estimates on $B_{r_{0} / 2}(q)$, i.e., $\left|u_{n}\right|_{C^{2, \alpha}} \leq C_{3}\left(\left|H_{n}\right|+\left|u_{n}\right|\right)$. Therefore, $u_{n}, \nabla u_{n}, \nabla^{2} u_{n}$ are uniformly bounded and equicontinuous.

As $\left\{\Sigma_{n}\right\}_{n \in \mathbb{N}}$ satisfy local area bounds, then $\left|\Sigma_{n} \cap B\left(r_{0} / 2\right)(y)\right| \leq C_{4}$. On the other hand, the monotonicity formula, Proposition 6.1, gives that $\left|\Sigma_{n}^{j} \cap B_{r_{0}}(y)\right| \geq$ $C_{5} r_{0}^{2}$, where $\Sigma_{n}^{j}$ is a connected component of $\Sigma_{n} \cap B_{r}$. It follows that the number of components of $\Sigma_{n} \cap B_{r_{0} / 2}(p)$ is finite and independent of $n$. By the Arzelà-Ascoli theorem we can extract a subsequence for which $\left\{u_{n}^{j}\right\}$ converges to $u$ for every $j$. Moreover, $u$ also satisfies the constant mean curvature equation (6.2). As the set of accumulation points of $\left\{\Sigma_{n}\right\}$ is compact in $B_{R}(p)$ we can cover this set by finite balls $B_{r_{0}}\left(p_{k}\right)$ with $k=1, \ldots, N$. Repeating the arguments in each of these balls and applying a diagonal argument we obtain a properly immersed surface $\Sigma$ on $B_{R}(p) \subset$ $M$ with constant mean curvature $H$. Since the surfaces $\Sigma_{n}$ are embedded, it follows that $\Sigma$ does not cross itself though it may have tangential self-intersections. Therefore, $\Sigma$ is properly weakly embedded in $M$.

Corollary 6.4 Let $\left(\Sigma_{n}, x_{n}\right) \subset\left(M_{n}, g_{n}, x_{n}\right)$ be a sequence of isoperimetric surfaces with $\left|A_{n}\right| \leq C$. Assume that $\left(M_{n}, g_{n}, x_{n}\right)$ converges, in the sense of CheegerGromov, to a three manifold $(M, g, x)$. There exists a properly embedded surface $\Sigma \subset(M, g, x)$ such that $\Sigma_{n} \rightarrow \Sigma$ in the sense of graphs and the convergence is with multiplicity one.

Sketch of the Proof First we remark that $\left\{\Sigma_{n}\right\}$ satisfy local area bounds. Indeed, $\operatorname{Area}\left(\Sigma_{n} \cap B_{r}(p)\right) \leq 2\left|\partial B_{r}(p)\right|$. By Proposition 6.3 we only need to rule out possibly multiplicities for the convergence $\Sigma_{n} \rightarrow \Sigma$ and points where $\Sigma$ fails to be embedded. 

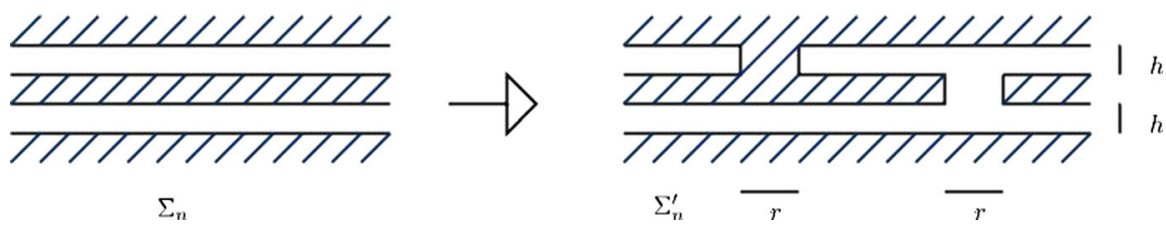

Fig. 2 Example of higher multiplicity
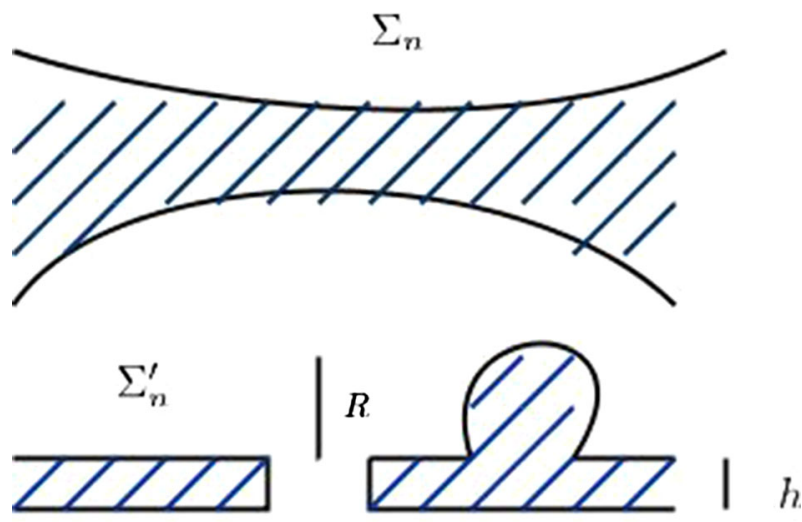

Fig. 3 Example of multiplicity two

If the multiplicity of the limit is bigger than two, then $\Sigma_{n} \cap B_{r}(p)$ has several components getting arbitrarily close. This allow us to do a local cut and past deformation, as shown in Fig. 2, that preserves the enclosed volume. If $\delta$ is the Euclidean metric, then $\frac{1}{\widetilde{C}} \delta \leq g_{n} \leq \widetilde{C} \delta$ and $\frac{1}{C^{\prime}}$ Area $_{\delta} \leq$ Area $_{g_{n}} \leq C^{\prime}$ Area $_{\delta}$. Thus, if $h \ll r$, then

$$
\operatorname{Area}_{g_{n}}\left(\Sigma_{n}^{\prime}\right) \leq \operatorname{Area}_{g_{n}}\left(\Sigma_{n}\right)-C_{1}^{\prime} r^{2}+C_{2}^{\prime} r h<\operatorname{Area}_{g_{n}}\left(\Sigma_{n}\right)
$$

The deformation needed for the multiplicity two case is shown in Fig. 3. The constraint on the enclosed volume implies that $\frac{4}{3} \pi R^{3} \approx \pi r^{2} h$. Hence, if $h \ll r$, then

$$
\operatorname{Area}\left(\Sigma_{n}^{\prime}\right) \leq \operatorname{Area}\left(\Sigma_{n}^{\prime}\right)-C_{1}^{\prime} r^{2}+C_{2}^{\prime} r h-C_{3}^{\prime} R^{2}+C_{4}^{\prime} R^{2}<\operatorname{Area}\left(\Sigma_{n}^{\prime}\right)
$$

The construction to deal with points where $\Sigma$ has tangential self intersections is similar to the multiplicity two case. The corollary now follows since these constructions contradict the fact that $\Sigma_{n}$ is isoperimetric for every $n$.

Theorem 6.5 (Korevaar-Kusner-Solomon [17]) If $\Sigma$ is a complete cmc surface properly embedded in a solid cylinder in $\mathbb{R}^{3}$, then $\Sigma$ is rotationally symmetric with respect to a line parallel to the axis of the cylinder. 
Theorem 6.6 (Da Silveira [11]) Let $\left(\Sigma, d s^{2}\right)$ be a complete orientable surface conformally equivalent to a compact Riemann surface punctured at a finite number of points. Let $L=\Delta+q$ be an operator on $\Sigma$ satisfying $q \geq 0$ and $q \not \equiv 0$. If $\Sigma$ has infinite area, then there exists a piecewise smooth function $f: \Sigma \rightarrow \mathbb{R}$ with compact support such that

$$
-\int_{\Sigma} f L f d_{\Sigma}<0 \text { and } \int_{\Sigma} f d_{\Sigma}=0 .
$$

\section{References}

1. Abresch, U., Rosenberg, H.: Generalized Hopf differentials. Mat. Contemp. 28, 1-28 (2005)

2. Almgren, F.J.: Existence and regularity almost everywhere of solutions to elliptic variational problems with constraints. Mem. Am. Math. Soc. 4(165), viii+199 (1976)

3. Barbosa, J.L., do Carmo, M.: Stability of hypersurfaces with constant mean curvature. Math. Z. 185, 339-353 (1984)

4. Barbosa, J.L., do Carmo, M., Eschenburg, J.: Stability of hypersurfaces with constant mean curvature in Riemannian manifolds. Math. Z. 197, 123-138 (1988)

5. Bavard, C., Pansu, P.: Sur le volume minimal de $R^{2}$. Ann. Sci. Ecole Norm. Sup. 19, 479-490 (1986)

6. Bray, H.: The Penrose inequality in general relativity and volume comparison theorems involving scalar curvature (thesis). (1998). arXiv:0902.3241v1

7. Bray, H., Morgan, F.: An isoperimetric comparison theorem for Schwarzschild space and other manifolds. Proc. Am. Math. Soc. 130(5), 1467-1472 (2002)

8. Burago, Y.D., Zagaller, V.A.: Geometric inequalities, volume 285 of Grundlehren der Mathematischen Wissenschaften [Fundamental Principles of Mathematical Sciences]. Springer, Berlin (1988) (Translated from the Russian by Sosinskir, A.B., Springer Series in Soviet Mathematics)

9. Chen, B.Y.: Geometry of Submanifolds. Pure and Applied Mathematics, vol. 22. Marcel Dekker, Inc., New York (1973)

10. Colding, T., Minicozzi, W.: A Course in Minimal Surfaces. Graduate Studies in Mathematics, vol. 121. American Mathematical Society, Providence (2011)

11. Da Silveira, A.: Stability of complete noncompact surfaces with constant mean curvature. Math. Ann. 277(4), 629-638 (1987)

12. Eichmair, M., Metzger, J.: Unique isoperimetric foliations of asymptotically flat manifolds in all dimensions. Invent. Math. 194(3), 591-630 (2013)

13. Gilbarg, D., Trudinger, N.S.: Elliptic Partial Differential Equations of Second Order. Fundamental Principles of Mathematical Sciences, vol. 224, 2nd edn. Springer, Berlin (1983)

14. Hauswirth, L., Pérez, J., Romon, P., Ros, A.: The periodic isoperimetric problem. Trans. Am. Math. Soc. 356(5), 2025-2047 (2003)

15. Hsiang, W.-Y.: On soap bubbles and isoperimetric regions in noncompact symmetric spaces. I. Tohoku Math, J. (2) 44(2), 151-175 (1992)

16. Hsiang, W.-T., Hsiang, W.-Y.: On the uniqueness of isoperimetric solutions and imbedded soap bubbles in noncompact symmetric spaces, I. Invent. Math. 98(1), 39-58 (1989)

17. Korevaar, N.J., Kusner, R., Solomon, B.: The structure of complete embedded surfaces with constant mean curvature. J. Differ. Geom. 30, 465-503 (1989)

18. Li, P., Yau, S.T.: A new conformal invariant and its applications to the Willmore conjecture and the first eigenvalue of compact surfaces. Invent. Math. 69, 269-291 (1982)

19. Marques, F.C., Neves, A.: Min-max theory and the Willmore conjecture. Ann. Math. 179(2), 683-782 (2014)

20. Morgan, F.: Regularity of isoperimetric hypersurfaces in Riemannian manifolds. Trans. Am. Math. Soc. 355, 5041-5052 (2003)

21. Morgan, F., Howe, S., Harman, N.: Steiner and Schwarz symmetrization in warped products and fiber bundles with density. Revista Matemática Iberoamericana 27(3), 909-918 (2011)

22. Morgan, F., Johnson, D.: Some sharp isoperimetric theorems for Riemannian manifolds. Indiana Univ. Math. J. 49(3), 1017-1041 (2000) 
23. Nardulli, S.: The isoperimetric profile of a smooth Riemannian manifold for small volumes. Ann. Glob. Anal. Geom. 36(2), 111-131 (2009)

24. Pedrosa, R.: The isoperimetric problem in spherical cylinders. Ann. Glob. Anal. Geom. 26(4), 333-354 (2004)

25. Pedrosa, R., Ritoré, M.: Isoperimetric domains in the Riemannian product of a circle with a simply connected space form and applications to free boundary problems. Indiana Univ. Math. J. 48(4), 13571394 (1999)

26. Petersen, P.: Riemannian Geometry. Graduate Texts in Mathematics, vol. 171, 2nd edn. Springer, New York (2006)

27. Ritoré, M.: Applications of compactness results for harmonic maps to stable constant mean curvature surfaces. Math. Z. 226(3), 465-481 (1997)

28. Ritoré, M., Ros, A.: Stable constant mean curvature tori and the isoperimetric problem in three space forms. Comment. Math. Helv. 67, 293-305 (1992)

29. Ritoré, M., Ros, A.: The spaces of index one minimal surfaces and stable constant mean curvature surfaces embedded in flat three manifolds. Trans. Am. Math. Soc. 348, 391-410 (1996)

30. Ros, A.: Compact hypersurfaces with higher order mean curvatures. Revista Matemática Iberoamericana 3, 447-453 (1987)

31. Ros, A.: The Willmore conjecture in the real projective space. Math. Res. Lett. 6, 487-493 (1999)

32. Ros, A.: The isoperimetric problem. In: Proceedings of Clay Research Institution Summer School (2001)

33. Schoen, R., Simon, L.: A new proof of the regularity theorem for rectifiable currents which minimize parametric elliptic functionals. Indiana Univ. Math. J. 31(3), 415-434 (1982)

34. Simon, L.: Lectures on geometric measure theory. In: Proceedings of the Centre for Mathematical Analysis, Australian National University (1983)

35. Topping, P.M.: Lectures on the Ricci flow, L.M.S. Lecture note series 325 C.U.P. (2006)

36. Torralbo, F., Urbano, F.: Compact stable constant mean curvature surfaces in homogeneous 3manifolds. Indiana Univ. Math. J. 61(3), 1129-1156 (2012)

37. White, B.: A local regularity theorem for mean curvature flow. Ann. Math. (2) 161(3), 1487-1519 (2005)

Publisher's Note Springer Nature remains neutral with regard to jurisdictional claims in published maps and institutional affiliations. 